Journal Club

Editor's Note: These short, critical reviews of recent papers in the Journal, written exclusively by graduate students or postdoctoral fellows, are intended to summarize the important findings of the paper and provide additional insight and commentary. For more information on the format and purpose of the Journal Club, please see http://www.jneurosci.org/misc/ifa_features.shtml.

\title{
Beyond Eye Movements: A Role for Frontal Eye Fields in Evaluating Decisions
}

\author{
(D) Chunyu A. Duan \\ Princeton Neuroscience Institute, Princeton University, Princeton, New Jersey 08540 \\ Review of Teichert et al.
}

Much research has been dedicated to understanding how we make decisions, but decision-making does not end with the execution of choice. A fundamental feature of decision-making is fallibility: we make and learn from our mistakes. Online evaluation of performance, especially after committing an error, provides an essential source of information for adjusting our strategy or updating our model of the external world.

Despite the ongoing debate about the exact cognitive mechanisms underlying error detection, work in humans and nonhuman primates has identified the medial prefrontal cortex (PFC) as a source of neural signals of performance monitoring (Carter et al., 1998; Stuphorn et al., 2000). In contrast, brain regions in the lateral PFC, such as the frontal eye fields (FEF), are thought to mediate motor planning and response selection but not postdecision evaluation. Neurons in the monkey FEF carry robust sensory, decision, and motor signals for selecting the appropriate response in oculomotor tasks (Kim and Shadlen, 1999). However, this may not be the whole story, because few stud-

Received March 27, 2014; revised April 20, 2014; accepted April 23, 2014. C.A.D. is supported by the Howard Hughes Medical Institute International Student Research Fellowship. I thank Jeffrey Schall, Matthew Botvinick, and Tobias Teichert for discussions regarding the reviewed paper; and Yael Niv, Carlos Brody, Timothy Hanks, Amitai Shenhav, and Jeffrey Erlich for helpful comments on this manuscript.

Correspondence should be addressed to Chunyu A. Duan, Princeton Neuroscience Institute, Princeton University, 184i Neuroscience Building, Princeton, NJ 08540. E-mail: cduan@princeton.edu.

DOI:10.1523/JNEUROSCI.1243-14.2014

Copyright $\odot 2014$ the authors $\quad 0270-6474 / 14 / 348061-02 \$ 15.00 / 0$ ies have looked at error-related signals in the FEF after response initiation. One important reason for the absence of reports linking FEF to performance monitoring is the difficulty of isolating error-related signals from concurrent sensorimotor activity.

A recent study published in The Journal of Neuroscience (Teichert et al., 2014) set out to fill this gap and investigate postdecision FEF activity using a categorization task designed to elicit errors. Two monkeys were trained to categorize the speed of a moving random dot pattern as either fast or slow by making a saccadic eye movement toward a green or red target. Task difficulty depended on the similarity of stimulus speed to the category boundary. To elicit behavioral errors, alternating blocks involved an unequal reward schedule that favored either the fast or the slow choice category. After the animals made a choice saccade, they were required to maintain fixation until they received a delayed auditory feedback, and then the reward itself. Although the animals were discouraged from "revising" their original decisions (since such trials were never rewarded), both animals reliably, although infrequently, corrected their errors even before the external feedback (Teichert et al., 2014, their Figs. 3B and 7). This behavioral result implies the existence of internally generated error signals, independent of external feedback. The authors thus set forth to investigate the neural correlates of error detection in this task. Indeed, Teichert et al. (2014) identified postsaccadic error-related signals before external feedback in one quarter of the FEF neurons they recorded.

To distinguish "performance monitoring” signals from alternative motor accounts such as saccade planning or execution, the authors conducted several control analyses. First, stimulus- and choice-related variables, including postdecision eye movements, were included as regressors in the linear regression model used to assess effects of error and difficulty on spike rate. Second, ipsilateral and contralateral errors were considered separately to test whether error signals depended on saccade direction. Postsaccadic activity in an oculomotor structure such as the FEF may simply reflect planning for corrective saccades to the contralateral side after ipsilateral errors. However, the authors found that most error cells responded to both ipsilateral and contralateral errors (Teichert et al., 2014, their Fig. 5), and further analyses focused only on these bilateral errorencoding cells.

Because animals were discouraged from corrective responses, errors could conceivably lead both to involuntary activation of error-correcting saccade planning signals and to enhanced activation in the opposite direction, to override the error-correcting activity. These corrective and anticorrective plans remain a potential motor account for bilateral error signals. To rule this out, the authors examined the activity of the same cells in a separate memory-guided saccade task. If 
both planning and inhibition of saccadic movement occur in these cells, similar activations should be seen during the memory period of the memory-guided task in which animals plan a saccade while withholding the action. Moreover, if the error response is due to this joint planning/ withholding response, parameter estimates for memory-period activity and error signals should be correlated. As this was not the case, the authors concluded that error-related signals in monkey FEF cannot be explained by motor hypotheses.

Testing the role of FEF in error detection requires careful examination of sensory- and motor-related activity. The endeavor exemplified by Teichert and colleagues (2014) argues against a segregation of the cognitive role of performance monitoring in medial PFC versus the motor role of response selection in lateral PFC. If the error-related signals observed in monkey FEF cannot simply be explained by motor planning, what are the underlying cognitive constructs? Teichert et al. (2014) provided support for several possible explanations, including the conflict monitoring account and the uncertainty account. Below I discuss the implications of these explanations in relation to an updated view of FEF's role in decision-making.

Conflict-monitoring theory (Botvinick et al., 2001) suggests that neurons monitor simultaneous activation of competing responses. This theory has been extended to explain error-related activity as a conflict between the incorrect response due to sensory/decision noise and the correct response following continued stimulus processing after committing to the error response (Yeung et al., 2004). This theory predicts that error-related signals should correlate with task difficulty. In support of the conflict-monitoring account, Teichert et al. (2014) saw a robust correlation across neurons between error and difficulty encoding (Teichert et al., 2014, Fig. 8). The conflict theory also predicts that difficulty-related signals should occur during decision formation while error-related signals should occur after the onset of error execution. The authors reported a trend of this timing difference (Teichert et al., 2014, Fig. 9); however, this did not hold up in a more conservative test that included only balanced error and difficulty cells.

Conflict signals have been observed consistently in human anterior cingulate cortex (ACC; Carter et al., 1998) and monkey supplementary eye field (SEF; Stuphorn et al., 2000). If the conflict theory of FEF error-related signals is borne out, it could imply a possible local taskspecific error signal that then feeds into medial areas such as SEF and ACC to trigger a more general conflict response. Alternatively, FEF error-related signals could represent an efference copy of the conflict response in $\mathrm{mPFC}$ that subsequently facilitates task-specific strategy adjustment in response selection. Further studies that simultaneously record from multiple cortical regions are needed to directly compare the timing of such conflict signals to identify the flow of activity underlying conflict monitoring (Rothé et al., 2011). Examining changes in behavior and $\mathrm{MPFC}$ activity during perturbation of FEF neurons immediately after response initiation can also differentiate the relative contributions of these areas.

Another important concept related to performance monitoring is decision uncertainty (Kepecs et al., 2008). Because error trials are associated with higher uncertainty, modulation of firing rate with errors does not differentiate true error signals from uncertainty signals. True error neurons increase their firing rates as behavioral error rate approaches $100 \%$, whereas cells encoding uncertainty fire maximally at chance performance. Teichert et al. (2014) found that even the strongest error-related signals were not associated with above-chance error likelihood (Teichert et al., 2014, their Fig. 10), supporting an uncertainty account of the error-related signals. However, because error modulation of firing rate was relatively small and animals rarely made mistakes, this analysis inherently favors the uncertainty account due to insufficient statistical power to reject it. One idea is to instead use the saccade-countermanding task, in which error rates are as high as $40 \%$, to test the uncertainty hypothesis. Interestingly, FEF neurons did not show error-related signals in a previous saccadecountermanding study (Hanes et al., 1998), where errors resulted from failures to withhold a prepared response. It is possible that FEF neurons selectively signal errors related to response selection, as demonstrated in Teichert et al.'s (2014) task, and not to errors due to failed inhibition.

In summary, Teichert et al. (2014) found signals related to postdecision evaluation in the FEF, suggesting an inte- grated circuit of performance monitoring and execution instead of a strict segregation of "actor" in lateral PFC and "critic" in medial PFC. Although the authors provided some evidence in favor of several cognitive theories to explain error detection, their data were not sufficient to accept or reject any of them. This reflects the ongoing debate regarding the cause and meaning of error signals. It is likely that FEF performs multiple functions in adaptive decision-making, especially since concepts such as response conflict and uncertainty are highly correlated. More importantly, if these error-related signals in the FEF actually contribute to how we learn from our mistakes, trial-by-trial variation of postdecision activity should correlate with the accuracy and reaction time of the following trial. Optogenetic perturbation of the FEF neurons immediately after response initiation could also provide insights to the causal role of FEF in online monitoring and adjustment of behavior.

\section{References}

Botvinick MM, Braver TS, Barch DM, Carter CS, Cohen JD (2001) Conflict monitoring and cognitive control. Psychol Rev 108:624-652. CrossRef Medline

Carter CS, Braver TS, Barch DM, Botvinick MM, Noll D, Cohen JD (1998) Anterior cingulate cortex, error detection, and the online monitoring of performance. Science 280:747-749. CrossRef Medline

Hanes DP, Patterson WF 2nd, Schall JD (1998) Role of frontal eye fields in countermanding saccades: visual, movement, and fixation activity. J Neurophysiol 79:817-834. Medline

Kepecs A, Uchida N, Zariwala HA, Mainen ZF (2008) Neural correlates, computation and behavioural impact of decision confidence. Nature 455:227-231. CrossRef Medline

Kim JN, Shadlen MN (1999) Neural correlates of a decision in the dorsolateral prefrontal cortex of the macaque. Nat Neurosci 2:176185. CrossRef Medline

Rothé M, Quilodran R, Sallet J, Procyk E (2011) Coordination of high gamma activity in anterior cingulate and lateral prefrontal cortical areas during adaptation. J Neurosci 31: 11110-11117. CrossRef Medline

Stuphorn V, Taylor TL, Schall JD (2000) Performance monitoring by the supplementary eye field. Nature 408:857-860. CrossRef Medline

Teichert T, Yu D, Ferrera VP (2014) Performance monitoring in monkey frontal eye field. J Neurosci 34:1657-1671. CrossRef Medline

Yeung N, Botvinick MM, Cohen JD (2004) The neural basis of error detection: conflict monitoring and the error-related negativity. Psychol Rev 111:931-959. CrossRef Medline 